\title{
BMJ Open Pancreatic resection with perioperative drug repurposing of propranolol and etodolac: trial protocol of the phase-II randomised placebo controlled PROSPER trial
}

To cite: Hüttner FJ, Rooman I, Bouche G, et al. Pancreatic resection with perioperative drug repurposing of propranolol and etodolac: trial protocol of the phase-II randomised placebo controlled PROSPER trial. BMJ Open 2020;10:e040406. doi:10.1136/ bmjopen-2020-040406

- Prepublication history for this paper is available online. To view these files, please visit the journal online (http://dx.doi org/10.1136/bmjopen-2020040406).

Received 12 May 2020 Revised 26 August 2020 Accepted 28 August 2020
D) Check for updates

(c) Author(s) (or their employer(s)) 2020. Re-use permitted under CC BY-NC. No commercial re-use. See rights and permissions. Published by BMJ.

For numbered affiliations see end of article.

\section{Correspondence to}

Dr Markus W Büchler; markus.buechler@med.uniheidelberg.de

\section{ABSTRACT}

Introduction Pancreatic cancer is the fourth-leading cause of cancer-related death in developed countries. Despite advances in systemic chemotherapy, the mainstay of curative therapy for non-metastatic disease is surgical resection. However, the perioperative period is characterised by stress and inflammatory reactions that can contribute to metastatic spread and disease recurrence. Catecholamines and prostaglandins play a crucial role in these reactions. Therefore, a drug repurposing of betablockers and cyclooxygenase inhibitors seems reasonable to attenuate tumour-associated inflammation by inhibiting psychological, surgical and inflammatory stress responses. This may cause a relevant antitumourigenic and antimetastatic effect during the perioperative period, a window for cancer-directed therapy that is currently largely unexploited.

Methods and analysis This is a prospective, singlecentre, two-arm randomised, patient and observer blinded, placebo-controlled, phase-II trial evaluating safety and feasibility of combined perioperative treatment with propranolol and etodolac in adult patients with nonmetastatic cancer of the pancreatic head undergoing elective pancreatoduodenectomy. 100 patients fulfilling the eligibility criteria will be randomised to perioperative treatment for 25 days perioperatively with a combination of propranolol and etodolac or placebo. Primary outcome of interest will be safety in terms of serious adverse events and reactions within 3 months. Furthermore, adherence to trial medication will be assessed as feasibility outcomes. Preliminary efficacy data will be evaluated for the purpose of power calculation for a potential subsequent phase-III trial. The clinical trial is accompanied by a translational study investigating the mechanisms of action of the combined therapy on a molecular basis.

Ethics and dissemination The PROSPER-trial has been approved by the German Federal Institute for Drugs and Medical Devices (reference number 4042875) and the Ethics Committee of the Medical Faculty of the University of Heidelberg (reference number AFmo-385/2018). The final trial results will be published in a peer-reviewed journal and will be presented at appropriate national and international conferences.
Strengths and limitations of this study

- This is the first clinical trial assessing a combined treatment with a betablocker (propranolol) and a cyclooxygenase inhibitor (etodolac) in the perioperative period surrounding partial pancreatoduodenectomy for cancer of the pancreatic head.

- Strengths of this study are its randomised, doubleblind, placebo-controlled design and its transparent design with the clear definitions of endpoints.

- Potential limitations include the rather small sample size leading to a limited power and the broad exclusion criteria, which may limit generalisability of the data, because of the selected trial population.

- If the trial treatment proves to be safe, the perioperative period may be used as a window for cancerdirected therapy and the results will form the basis for a subsequent phase-III trial.

Trial registration numbers DRKS00014054; EudraCT number: 2018-000415-25.

\section{INTRODUCTION}

More than 450000 new cases of pancreatic cancer are diagnosed worldwide per year. ${ }^{1}$ In contrast to many other cancers, its incidence and mortality have been rising during the last decades and the prognosis is devastating with a 5-year overall survival of $<10 \%{ }^{23}$ Despite substantial advancements in systemic chemotherapy during recent years, the mainstay of curatively intended treatment remains surgical resection. However, only a small proportion of patients present with potentially resectable disease (primary resectable or borderline resectable) at diagnosis. ${ }^{4}$ With modern multimodal treatment strategies consisting of resection and adjuvant chemotherapy, a median overall survival of 54 
months can be reached, but disease-free survival at 3 years is still $<50 \%$ in these patients. ${ }^{5}$

For patients with potentially resectable tumours, the perioperative time period, which spans an interval from the time point of diagnosis until the actual start of adjuvant chemotherapy, represents a window of opportunity for perioperative therapy aimed to reduce recurrence. ${ }^{6}$ The psychological stress and the emotions around diagnosis, surgery and upcoming chemotherapy are accompanied by increased release of catecholamines exerting their effects on tumour cells and stromal cells that express adrenergic receptors. On one hand, catecholamine release is an adaptive reaction to cope with stressful situations by modulating the immune response and mobilising metabolic reserves. On the other hand, these stress responses are also pro-tumourigenic with pleiotropic effects on the primary tumour, the tumour microenvironment, malignant cells in the circulation and on pre-existing micrometastases. ${ }^{7}$ Interestingly, the effects of catecholamines may be counteracted by adrenergic receptor antagonists (beta-blockers), often prescribed for hypertension or cardiac disease.

Surgically induced inflammation and the subsequent release of prostaglandins are additional mediators of tumourigenic and prometastatic effects in the perioperative period despite their role in tissue repair and regeneration. ${ }^{8}$ Prostaglandins facilitate tumour cell survival, proliferation and invasion and also counteract immunosurveillance. ${ }^{9-11}$ Prostaglandins promote angiogenesis and epithelial-mesenchymal transition and they lead to a release of prosurvival and growth factors for malignant cells. ${ }^{78}$ In addition, prostaglandins promote microvascular barrier dysfunction, endothelial hyperpermeability and lymphatic dilation, which can facilitate lymphatic and distant metastases. ${ }^{12-14}$ Prostaglandin synthesis from arachidonic acid involves the cyclooxygenases (COX), of which COX-2 is the inducible form that is particularly active during inflammation by generating prostaglandin E2 (PGE-2). COX are druggable targets and the abovementioned effects could be counteracted by selective COX-2 inhibitors.

The term 'drug repurposing' describes an approach trying to repurpose well-characterised drugs for a previously untested indication. This emerging strategy is of particular interest for oncological purposes. ${ }^{15} 16$ In terms of drug repurposing a combination therapy of propranolol, a non-selective beta-blocker and etodolac, a semiselective COX-2 inhibitor, in the perioperative setting of pancreatic cancer resection may result in attenuation of tumour-associated inflammation and stress responses. ${ }^{17}$ By these mechanisms, it may cause a relevant antitumourigenic and antimetastatic effect during the perioperative period, which is a currently largely unexploited window for therapies aiming to influence these stress and inflammatory responses. Promising results of this 'drug repurposing' have been shown in both, preclinical and early clinical trials for other solid cancer entities. In an experimental liver metastasis mouse model of colorectal cancer, treatment with propranolol and etodolac reduced hepatic metastases in the context of surgery. ${ }^{18}$ Furthermore, in a phase-II randomised controlled trial on colorectal cancer, the treatment was well tolerated and showed favourable results regarding early biomarkers of metastatic potential, but also 3-year recurrence rates. ${ }^{19}$

The primary objective of this pilot trial will be to assess the safety of the combination therapy with propranolol and etodolac in the perioperative setting of pancreatic cancer surgery. Beyond safety, feasibility will be analysed, since the two predominant factors for curative treatment (surgery and adjuvant chemotherapy) must not be affected by the above-mentioned drug administration. If the trial treatment proves to be safe and feasible, the survival data derived from the current trial will form the basis for a subsequent confirmatory phase-III trial assessing its efficacy.

\section{METHODS AND ANALYSIS \\ Study design}

The PROSPER trial is a two-arm randomised, patient and observer blinded, placebo-controlled, phase-II trial. The primary objective is to evaluate safety and feasibility of perioperative propranolol and etodolac treatment in patients with resectable cancer of the pancreatic head planned for elective pancreatoduodenectomy. Additionally, early parameters of efficacy will be assessed by analysing survival of the patients and by an ancillary translational study investigating the mechanisms of action of the combined therapy on a molecular basis. The trial will be performed in a single-centre setting at the Department of General, Visceral and Transplantation Surgery of the University Hospital Heidelberg, Germany, a centre of excellence for pancreatic surgery with high volume in oncological pancreatic surgery. Before inclusion of the first subject, the trial was registered with the German Clinical Trials Register (DRKS00014054). The full protocol and the current protocol publication were prepared in line with the Standard Protocol Items: Recommendations for Interventional Trials (SPIRIT) recommendations. ${ }^{20}$

The two drugs under investigation, propranolol and etodolac, will be repurposed in the perioperative setting of patients undergoing elective pancreatic head resection in order to potentially improve oncological outcome by suppressing several pathways of systemic stress and inflammatory responses.

Eligible patients, who have provided written informed consent (for a model consent form see online supplemental file 1), will be randomised to either the combined drug therapy with propranolol and etodolac, starting 10 days preoperatively until postoperative day (POD) 14, or corresponding placebo treatment. Pancreatic resection and postoperative care will be performed according to local standards of the Department of General, Visceral 


\section{Box 1 Major eligibility criteria of the trial}

\section{Inclusion criteria:}

- Resectable malignancy of the pancreatic head, eligible for elective pancreatoduodenectomy in curative intent.

- WHO/Eastern Cooperative Oncology Group (ECOG) performance status $0-2$.

- Age $\geq 18$ years.

- American Society of Anesthesiologists (ASA) score I-III.

- Patient must be able to understand the consequences of trial participation and to provide written informed consent.

- Written informed consent from the trial subject has been obtained.

- Female subject must be postmenopausal (for at least 6 months), surgically sterile, abstinent, or, if sexually active, be practising an effective method of birth control (eg, prescription of oral contraceptives, contraceptive injections, intrauterine device, double-barrier method, contraceptive patch, male partner sterilisation) before entry and throughout the study; and have a negative serum human choriongonadotropin (B-hCG) pregnancy test at screening.

\section{Exclusion criteria}

- Any contraindication for pancreatoduodenectomy.

- Metastatic disease (stage IV).

- Patients scheduled for palliative resection (no curative treatment intention).

- Patients scheduled for extended resections (ie, arterial resections, planned multivisceral resections).

- Acute or ongoing episode of cholangitis: fever and pain in the right upper quadrant of the abdomen together with increased infectious parameters and elevated values (alkaline phosphatase, gamma glutamyl transpeptidase) $>3 x$ upper limit of norm (ULN).

- Acute or ongoing episode of pancreatitis (clinical symptoms of pancreatitis, increased lipase $>3 \times$ ULN and/or C reactive protein values, radiological or intraoperative signs of acute pancreatitis).

- Chronic neuropathy $>$ grade 2.

- Renal failure, measured by glomerular filtration rate (GFR) $<50 \mathrm{~mL} /$ $\mathrm{min} / 173 \mathrm{~m}^{2}$ (calculated according to the Chronic Kidney Disease Epidemiology Collaboration (CKD-EPI) formula).

$\rightarrow$ Known liver cirrhosis of any grade.

- Atrioventricular block.

- Pregnant or breastfeeding women.

- Mental or organic disorders, which could interfere with giving informed consent or receiving treatments.

- Any contraindication to propranolol and/or etodolac. *

*For a list of all contraindications see online supplemental file 2.

and Transplantation Surgery of the University Hospital Heidelberg. ${ }^{21}$

\section{Study population and eligibility criteria}

Patients with malignancy of the pancreatic head scheduled for elective pancreatoduodenectomy in curative intent will be consecutively screened for eligibility according to the inclusion and exclusion criteria that are listed in box 1. Eligible patients will be informed verbally and in writing in comprehensible language about the nature, scope and possible consequences of the trial by a trial investigator. If a patient has provided written informed consent for trial participation (s) he will be randomised to the verum or placebo group.
Ineligible patients and those who refused to participate will be documented in a screening log with the respective reason for non-participation.

\section{Subject withdrawal}

Patients are free to leave the trial at any time and without giving reasons for their decision. Subjects may be withdrawn from the trial for the following reasons:

A. At their own request.

B. If, in the investigator's opinion, continuation of the trial would be detrimental to the subject's well-being, for example, symptomatic bradycardia after start of treatment, allergic reactions to trial treatment. In case of (B), the reason for withdrawal must be recorded in the case report form (CRF) and in the patient's medical records.

Patients, who withdrew or were withdrawn from the trial, will not be replaced.

\section{Sample size}

Since the current trial is conducted in an exploratory stage and not confirmatory, no formal sample size calculation was performed. The sample size of 40 patients per group was judged to be sufficient for an assessment of safety and feasibility by a panel of clinical and methodological experts at the investigators' institution. All analyses will be interpreted as exploratory. Considering a drop-out rate of approximately $20 \%$, a total of 100 patients will be randomised within the trial. The estimated patient flow within the trial is depicted in figure 1.

\section{Trial procedures}

\section{Perioperative combination therapy}

There will be one experimental arm (A) and one control arm (B):

A. Propranolol+etodolac.

B. Placebo.

\section{ARM A}

Oral intake of propranolol $2 \times 20 \mathrm{mg} /$ day for 10 days preoperatively, $2 \times 40 \mathrm{mg}$ for the day of surgery and 1 week after surgery, $2 \times 20 \mathrm{mg}$ for the second postoperative week combined with oral intake of etodolac $2 \times 400 \mathrm{mg} /$ day for a total of 25 days perioperatively (starting at 10 days preoperatively). The dosage is summarised in table 1 .

\section{ARM B}

Oral intake of placebo tablets/capsules of the same appearance, size and weight as propranolol and etodolac. Placebo will be taken $2 \mathrm{x}$ /day for a total of 25 days perioperatively (starting at 10 days preoperatively).

With regard to current evidence and personal communications with experts on the combined administration of propranolol and etodolac, we propose to use the dose of etodolac that was used by Bhattacharyya $e t a l$ in their clinical trial in metastatic PDAC and in the perioperative trial in colorectal cancer of Haldar et al. ${ }^{19}{ }^{22}$ Propranolol will be used in a standard dose of $2 \times 20 \mathrm{mg}$, which was also used by Haldar et al in their colorectal cancer trial and an 


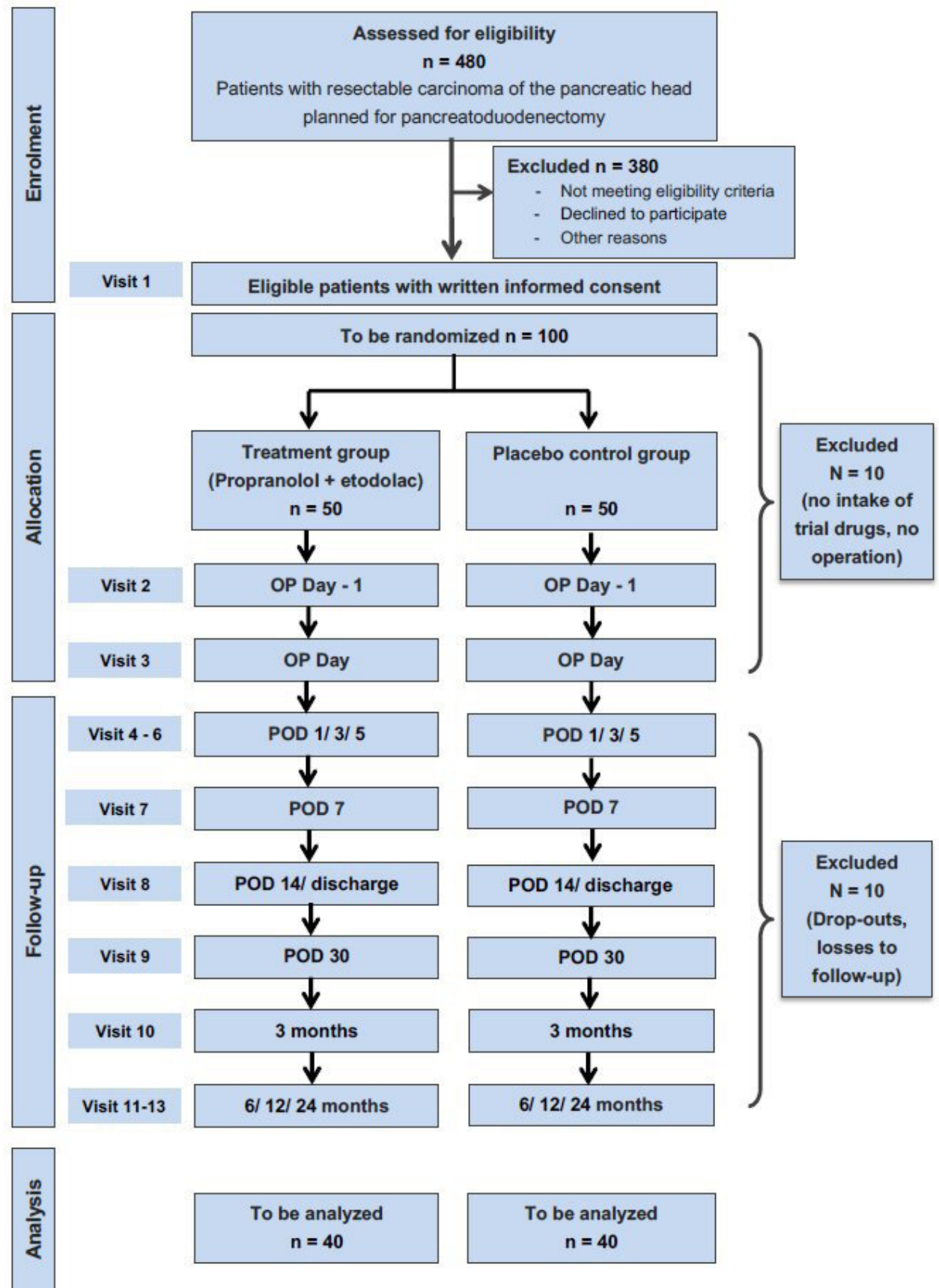

Figure 1 Trial flow chart. n,number; OP, operation, surgical intervention; POD, postoperative day.

increased dose of $2 \times 40 \mathrm{mg}$ on the day of surgery and the first postoperative week due to the increased stress factors in this period. ${ }^{19}$

Bhattacharyya et al observed no treatment-associated serious adverse events (SAEs). ${ }^{22}$ Equally, in the perioperative colorectal cancer trial the combination therapy appeared safe with only one case of symptomatic bradycardia that led to exclusion of the patient and two cases of tolerable bradycardia. There were no differences in the rate of postoperative AEs between the treatment and the placebo groups.

No dose adjustments are planned in individual subjects. In cases that require a temporary discontinuation of trial treatment (eg, swallowing of tablets not possible due to severe gastroparesis; treatment on intensive care unit with, eg, catecholamine therapy; etc), the treatment will be paused and resumed as soon as possible if reasonable. The percentage of the finally taken medication from the planned medication will be documented in the CRF (adherence).

In case of any acute deterioration of health by any cause, the patients can and will be treated by any appropriate measures without restrictions.

\section{Surgery and biospecimen collection}

Partial pancreatoduodenectomy will be performed according to local standards at the Department of General, Visceral and Transplantation Surgery of the 


\begin{tabular}{lll}
\hline $\begin{array}{l}\text { Table } 1 \text { Dosage of trial medication } \\
\text { Time point }\end{array}$ & Morning & Evening \\
\hline 10 Preop. Days & P $20 \mathrm{mg}$ & P $20 \mathrm{mg}$ \\
& E $400 \mathrm{mg}$ & E $400 \mathrm{mg}$ \\
Day of surgery & P $40 \mathrm{mg}$ & P $40 \mathrm{mg}$ \\
& E 400 mg & E 400 mg \\
POD 1-7 & P $40 \mathrm{mg}$ & P $40 \mathrm{mg}$ \\
POD 8-14 & E 400 mg & E 400 mg \\
& P $20 \mathrm{mg}$ & P $20 \mathrm{mg}$ \\
\hline
\end{tabular}

$\mathrm{E}$, etodolac or corresponding placebo; P, propranolol or corresponding placebo; POD, postoperative day.

Heidelberg University Hospital. ${ }^{21}$ Postoperative care consists of a fast-track concept including a no-drain policy in routine cases, early mobilisation and early oral feeding.

For the translational part of the trial, peripheral blood samples will be collected during the screening visit, the day before surgery, the day of surgery, on POD 1, 3, 14 (or day of discharge) and 3 months postoperatively. Additionally, portal venous blood will be taken intraoperatively immediately after dissection of the hepatoduodenal ligament and before resection of the tumour. Immediately after resection, a biopsy of $0.5-1 \mathrm{~cm}^{3}$ will be excised from the resected specimen for biobanking and molecular analysis in the translational part of the study. Detailed methodology of the translational study will be described in a separate publication.

\section{Trial endpoints}

\section{Safety endpoints}

Safety will be evaluated by the rates of SAE and serious adverse reactions (SAR) in the two treatment groups within a period of 3 months postoperatively.

Further measurements of safety include postoperative mortality within 30 and 90 days, pancreas-associated morbidity, such as postoperative pancreatic fistula according to the up-dated International Study Group of Pancreatic Surgery (ISGPS) definition, ${ }^{23}$ delayed gastric emptying and postpancreatectomy haemorrhage according to the respective ISGPS definitions, ${ }^{24}{ }^{25}$ postoperative biliary leakage according to the ISGLS definition $^{26}$ and postoperative intra-abdominal fluid collection or abscess.

\section{Feasibility endpoints}

Feasibility will be assessed by the evaluation of adherence to study medication intake. The rationale for this endpoint is that there are some factors in the perioperative period, which might preclude patients from taking the intended medication (eg, delayed gastric emptying/ gastroparesis, nausea/vomiting, intolerable bradycardia under propranolol, etc). This would hamper a widespread implementation into treatment protocols. Adherence to study medication will thus provide more insight into the feasibility of implementing treatment with propranolol and etodolac into perioperative treatment plans. Adherence to trial medication will be documented in a medication diary. Every patient will be asked to document his drug intake or any impediments. Furthermore, all used or unused blister packs will need to be turned in to the trial centre by the patients. Opened packages with left-over investigational medicinal products (IMPs) will be accounted for at the trial centre. The percentage of the finally taken medication from the planned medication will be documented in the electronic CRF (eCRF).

Furthermore, completion of adjuvant chemotherapy will be evaluated as another measure of feasibility. This endpoint provides further insight into the practicality of implementing the trial intervention into modern routine treatment schedules.

\section{Efficacy endpoints}

Overall and disease-free survival will be assessed as oncological long-term variables. Survival is the ultimate goal of any cancer directed therapy. Apart from overall survival, disease-free survival has been proven to be a reliable parameter in phase II/III clinical trials.

Furthermore, rates of local and distant recurrence will be assessed as another efficacy outcome. All of these preliminary efficacy endpoints will be evaluated for the purpose of a power calculation for a potential subsequent phase-III trial.

\section{Biological endpoints}

Biological endpoints/biomarkers will be measured at different time points of study conduct as described above.

The following parameters will be assessed:

Blood samples:

1. Routine laboratory biomarkers: differential blood count, CRP, albumin, carbohydrate antigen 19-9 (CA 19-9), carcinoembryonic antigen.

2. Translational study biomarkers: phenotyping of circulating immune cells (fluorescence-activated cell sorting (FACS) ), cytokine multiplex, PGE-2 levels, circulating tumour cells, RNA sequencing in periphery blood cells (leucocytes).

Tissue samples (translational part of the study):

1. Biomarkers associated with COX-2 inhibition: COX-2, PGE-2, interleukin 6 (IL-6) expression.

2. Biomarkers associated with $\beta$-blockade: adrenoceptor beta 2, vascular endothelial growth factor, matrix metalloproteinase 2 (MMP2), MMP4, S100, brainderived neurotrophic factor expression.

3. Characterisation of the immune cell infiltrate.

4. RNA sequencing of bulk tissue or of sorted tumour and stromal cells.

In addition, tumour cells will be cultures as organoids for further biological substudies.

Table 2 gives an overview of the clinical trial visits and displays which endpoints will be captured during the respective visits. 
Table 2 Trial visits and assessment of endpoints

\begin{tabular}{|c|c|c|c|c|c|c|c|c|c|}
\hline & Visit 1 & Visit 2 & Visit 3 & Visit 4-6 & Visit 7 & Visit 8 & Visit 9 & Visit 10 & Visit 11-13 \\
\hline Documentation & Screening & $\begin{array}{l}\text { Day } \\
\text { before } \\
\text { surgery }\end{array}$ & $\begin{array}{l}\text { Day of surgery } \\
10-28 \text { days } \\
\text { after } V 1\end{array}$ & $\begin{array}{l}\text { POD } \\
1 / 3 / 5\end{array}$ & POD 7 & $\begin{array}{l}\text { POD 14/ } \\
\text { discharge }\end{array}$ & POD 30 & 3 months & $\begin{array}{l}6 / 12 / 24 \\
\text { months }\end{array}$ \\
\hline Eligibility criteria & $x$ & & $x$ & & & & & & \\
\hline $\begin{array}{l}\text { Baseline data, } \\
\text { demographics }\end{array}$ & $x$ & & & & & & & & \\
\hline Previous medication & $x$ & & & & & & & & \\
\hline Assessment of safety & & $x$ & $x$ & $x$ & $x$ & $x$ & $x$ & $x$ & \\
\hline Feasibility/adherence & & $x$ & $x$ & $x$ & $x$ & $x$ & $x$ & $x$ & \\
\hline Secondary endpoints* & & & $x$ & $x$ & $x$ & $x$ & $\mathrm{x}$ & & \\
\hline Survival/recurrence & & $x \dagger$ & $x \dagger$ & $x \dagger$ & $x \dagger$ & $x+$ & $x \dagger$ & $x$ & $x$ \\
\hline Tissue sampling & & & $x$ & & & & & & \\
\hline
\end{tabular}

*Details of surgery, pathological results (TNM stage), pancreas specific complications.

†Only survival.

łBlood count, CRP, creatinin, bilirubin, albumin, international normalised ratio, CEA, CA 19-9 (visit 1 and/or 2, 10), pregnancy test in case of childbearing potential (visit 1).

§POD 1 and 3 only.

CA, carbohydrate antigen; CEA, carcinoembryonic antigen; CRP, C reactive protein; POD, postoperative days.

\section{Safety objectives and assessment of safety}

All patients will be closely monitored for the occurrence of AEs. In this trial, all AEs will be documented from the first administration of the IMPs (10 days prior to surgery) until 3 months postoperatively. An $\mathrm{AE}$ is any untoward medical occurrence in a trial subject administered an IMP. There does not necessarily have to be a causal relationship with the IMP or any other trial-related procedure. An SAE or SAR is any untoward medical occurrence, without or with a reasonably possible causal relationship with the IMP, that at any dose:

1. Results in death.

2. Is life-threatening at the time of the event.

3. Requires inpatient hospitalisation or prolongation of existing hospitalisation.

4. Results in persistent or significant disability/incapacity.

5. Is a congenital anomaly or birth defect.

6 . Is, in the opinion of the investigator, otherwise medically relevant (eg, requiring relaparotomy, endoscopic treatment or interventional percutaneous drainage, etc).

Pre-existing diseases are not documented as AE but as concomitant diseases. New diseases or deteriorations of preexisting illnesses are also an $\mathrm{AE}$ in the context of this clinical trial. However, a preexisting disease that leads to a treatment measure, which has already been planned before the start of the clinical trial, is not regarded as an $\mathrm{AE}$ or SAE. Furthermore, changes of a pre-existing disease, which represent the natural/expectable course of the disease are not regarded an AE. Routine medical treatment after pancreatoduodenectomy will not be regarded as $\mathrm{AE}$. In the postoperative period, expectable clinical and/or laboratory findings (eg, postoperative pain, delayed reuptake of bowel function, increase of CRP and white blood cells, decrease of haemoglobin value, increase of liver function parameters, etc) will not be regarded as (S) AE as long as they are without deviation from the expectable clinical course. A list of the expectable changes is included in the full trial protocol and can be obtained on request from the corresponding author.

The data safety monitoring board will receive listings of all SAE after the accrual of 20, 40 and 60 patients for review and assessment of patient risk and potential differences between the trial groups.

The Coordination Center for Clinical Trials (KKS) Heidelberg will be responsible for pharmacovigilance and will provide a development safety update report once a year prepared according to the International Council for Harmonisation (ICH) guideline E2F.

\section{Ancillary translational study}

In addition to routine blood sampling, which includes blood count, CRP, creatinine, bilirubin, albumin and international normalised ratio, blood samples for translational tests will be taken at the above-mentioned points of time. The translational tests will consist of a cytokine multiplex (screening visit and day before surgery), PGE-2 
levels (screening visit and day before surgery), circulating tumour cells (POD 3), RNA sequencing in periphery blood cells (leukocytes; POD 3). The tumour tissue, which is sampled at surgery, will be investigated by tissue cultures (organoids and pluripotent stem cells). Furthermore, COX-2 expression, PGE-2 levels and immune cell infiltration will be investigated and RNA sequencing of bulk tissue or of sorted tumour and stromal cells will be performed.

\section{Data handling and monitoring}

An investigator or a designated representative will enter all protocol-required data into the eCRF. All entries in the eCRF must be verifiable by source documents. Data entries will undergo an automatic online check for plausibility and consistency. On completion of the eCRF, the investigator has to confirm the accuracy of all data by signing sections online in the eCRF.

On site visits will be done by the monitor at regular intervals to ensure compliance with the trial protocol, ICH Good Clinical Practice (GCP) guidelines and legal aspects. The monitor must be given access to all trial relevant documents by the investigator. He will review the entries into the eCRF for completeness and correctness and verify the entries on the basis of the source documents. The responsible monitor and the data manager can generate special questions (queries) that will be sent back to the responsible investigator. The investigator or a designated representative will have to answer them all in a timely manner. Data management and monitoring activities will be performed according to the current standard operating procedures of the KKS or the Study Center of the German Surgical Society (SDGC), respectively.

\section{Statistical analysis}

The main safety, feasibility and efficacy variables will be analysed with generalised linear models or Cox regression models. Parameter estimates from these models will be reported with $95 \%$ profile likelihood-based CIs. All other variables will be tabulated by treatment group using number of missing and non-missing values, the quartiles, mean and SD.

All subjects who have ever been randomised to study treatment will form the full analysis set for efficacy purposes. All patients will be analysed according to the treatment group they have been randomised to, regardless of the treatment they actually received (intention to treat). A restricted full analysis set will be formed by the set of subjects undergoing surgical resection of the pancreas. Patients not undergoing surgery at all or patients, who did not take at least $50 \%$ of their preoperative trial medication will be replaced. The per-protocol set will comprise all patients receiving at least $75 \%$ of study medication at the appropriate time points. The safety set will contain all patients ever receiving study medication and will assign patients to treatment group according to the treatment they actually received.
Analysis with respect to the primary safety endpoints will be conducted using the safety set. Feasibility will be compared using the full analysis set, whereas analysis on efficacy will be conducted using the restricted full analysis set. A sensitivity analysis for efficacy will be per-formed for the per-protocol set.

The rate of SAE/SAR will be used as the primary safety variable. It will be analysed using a Poisson regression model using the time under risk as an offset and treatment group as an explanatory variable.

\section{Methods for minimising bias \\ Minimising selection bias}

Patients will be consecutively screened and all eligible patients will be asked for informed consent. All patients who have been screened but not included into the trial, will be documented together with the respective reason for exclusion to assure transparency regarding patient selection. Included patients will be allocated concealed by $1: 1$ randomisation using a random list. Block randomisation of variable sizes will be performed.

The pharmacy will manufacture consecutively numbered packages of trial medication of either verum or placebo according to the random list and deliver these to the trial centre on demand. On inclusion of a new subject, the investigator or designated representative (eg, a study nurse) will hand out the package with the lowest remaining number to the patient and inform the monitoring about every new randomisation.

\section{Minimising performance and detection bias}

Patients as well as all trial will be blinded with regard to the trial drugs by means of placebo drugs in the control group. Since standard treatment is no specific perioperative medication and no proven treatment during this period exists, which may improve survival, the use of placebo is justified. Therefore, patients with placebo treatment will not be at risk of harm.

If it is medically imperative to know whether the patient receives verum or placebo, the investigator or an authorised person should break the randomisation code for the concerned patient. The investigator or the person, who breaks the blind, must record the date and the reason for unblinding in the subject's medical record and in the randomisation tool. Whenever possible, the principal investigator should be contacted before the blind is broken.

\section{Minimising attrition and reporting bias}

The trial will be reported according to the Consolidated Standards of Reporting Trials statement ${ }^{27}$ and to minimise reporting bias the trial was registered in advance in the German Clinical Trials Registry DRKS (DRKS00014054), an approved Primary Register of the WHO International Clinical Trials Registry Platform meeting the requirements of the International Committee of Medical Journal Editors. For transparency regarding conduct and 
reporting of this trial, the protocol is hereby published according to the SPIRIT statement. ${ }^{20}$

\section{Ethics and regulatory aspects}

Ethical basis for this trial are the principles described in the applicable version of the Declaration of Helsinki. ${ }^{28}$ The present trial is conducted in accordance with the internationally recognised ICH-GCP, national regulatory requirements like the German Drug Law (AMG) and German GCP Regulation, as well as the European regulations Directive 2001/20/EC and Commission Directive 2005/28/EC.

Before start of the clinical trial, the trial was registered with EudraCT (2018-000415-25) and in the German Clinical Trials Registry (DRKS00014054). The trial was approved by the competent authority, the German Federal Institute for Drugs and Medical Devices (reference number 4042875 on 28 August 2018). Furthermore, the trial was reviewed by the Ethics Committee of the Medical Faculty of the University of Heidelberg and was positively evaluated (reference number AFmo-385/2018 on 10 August 2018).

Data protection laws (Bundesdatenschutzgesetz and Landesdatenschutzgesetz Baden-Württemberg) as well as the provisions of the General Data Protection Regulation (Regulation 2016/679 of the European Union) will be respected. It is assured by the sponsor that all investigational materials and data will be pseudonymised in accordance with data protection legislation before scientific processing.

Trial drugs are licensed within the EU and dosage for each drug lies within the licensing recommendations. For the combination propanolol/etodolac data have been published documenting good tolerance. Exclusion criteria will guarantee that patients with pre-existing severe diseases are excluded. Therefore, the risk-benefit ratio for this selected patient group is positive.

Patients may not be enrolled into the present trial unless they have consented to take part in the trial after having been informed verbally and in writing in comprehensible language of the nature, scope and possible consequences by a trial investigator. Together with the consent to take part in the trial, the trial subject must also agree to representatives of the sponsor (eg, monitors or auditors) or the competent supervisory or federal authorities having access to the data recorded within the framework of the clinical trial. The trial subject will be informed of the potential benefit and possible side effects of the IMP and placebo, and of the need and reasons to conduct a placebo controlled clinical trial. It must be clear to trial subjects that he or she can withdraw his or her consent at any time without giving reasons and without jeopardising his/her further course of treatment.

\section{Amendments to the trial protocol}

Amendments made in accordance with $\S 10$ Secs. 1 and 4 GCP Regulations that require approval are submitted to the ethics committee and the federal higher authority and will not be implemented until approved.

Since the start of the PROSPER trial, two amendments have been implemented and have been approved. These included two relevant changes to the eligibility criteria: the exclusion criterion 'preoperative CA 19-9 >400 U/mL' was deleted during the first amendment and the exclusion criterion. 'Previous neoadjuvant therapy' was deleted during the second amendment. This included the addition of a subgroup analysis regarding efficacy of patients with versus without neoadjuvant therapy. Furthermore, details on neoadjuvant treatment (treatment protocol, dosage, etc) will be documented together with the baseline characteristics on inclusion into the trial. Additionally, the amendments comprised only minor changes and precisions, for example, regarding the amount of blood sampled during the translational tests.

\section{Patient and public involvement}

Patients or the public were not directly involved in the development of this trial protocol. However, our group conducted a priority setting partnership on pancreatic cancer identifying the most important research questions by a priority setting group composed of patients, caregivers, members of patient support groups and healthcare professionals. The generated top ten list of research priorities included several aspects that are addressed by this trial, for example, 'How can the best treatment for each individual patient with PC be identified?'. ${ }^{29}$ Additionally, it is planned to distribute the results of this trial after its completion within patient support groups such as the German 'Arbeitskreis der Pankreatektomierten e.V.'.

\section{Dissemination}

It is planned to publish the trial results of this trial in a scientific, peer-reviewed journal and to present the results at appropriate national or international scientific conferences.

Results from the accompanying translational part of the study will be published in mutual agreement with the principal investigator of the clinical trial and the responsible investigator of the translational study.

A full deidentified individual patient dataset of the trial will be made available after trial completion and publication on reasonable request from the corresponding author.

\section{Author affiliations}

${ }^{1}$ Department of General, Visceral and Transplantation Surgery, University of Heidelberg, Heidelberg, Germany

${ }^{2}$ The Study Center of the German Surgical Society (SDGC), University of Heidelberg, Heidelberg, Germany

${ }^{3}$ The Anticancer Fund, Brussels, Belgium

${ }^{4}$ Laboratory of Medical and Molecular Oncology, Vrije Universiteit Brussel, Brussel, Belgium

${ }^{5}$ Coordination Center for Clinical Trials, Heidelberg University, Heidelberg, Germany

Acknowledgements We thank Inga Rossion (SDGC) and Liese Vandeborne (Anticancer Fund) for their ongoing support regarding this trial in various aspects. Additionally, we appreciate the work of all contributors from the Anticancer Fund, 
the KKS, the SDGC as well as from the clinical study center of the Department of the General, Visceral and Transplantation Surgery of the University Heidelberg. Furthermore, we want to acknowledge the honorary work of the members of the DSMB: Richard Jackson, Cancer Research UK Liverpool Cancer Trials Unit, University of Liverpool, Liverpool, UK; Paul Karanicolas, Sunnybrooke Health Sciences Centre, Toronto, Ontario, Canada; Erica Sloan, Institute of Pharmaceutical Sciences, Monash University, Parkville, Victoria, Australia.

Contributors FJH, IR, GB, PK, JH, AM, TH, OS and MKD designed the study protocol. All authors contributed to the study design. FJH, IR, GB, OS, MWB and MKD initiated this clinical study. FJH and MKD wrote the paper. All authors approved the final version of this manuscript. All authors agreed to be accountable for all aspects of the work, and ensure that any questions related to the accuracy or integrity of any part of the work will be appropriately investigated and resolved.

Funding This clinical trial is financed with donations of Het Anti-Kankerfonds (Anticancer Fund), a Belgian-based private foundation (grant number: A56). № commercial stakeholder was involved in the planning or conduct of this trial.

Disclaimer The funding source of the trial does not have a role in data collection, data analysis, or interpretation of the trial results.

Competing interests None declared.

Patient and public involvement Patients and/or the public were not involved in the design, or conduct, or reporting, or dissemination plans of this research.

Patient consent for publication Not required.

Provenance and peer review Not commissioned; externally peer reviewed.

Open access This is an open access article distributed in accordance with the Creative Commons Attribution Non Commercial (CC BY-NC 4.0) license, which permits others to distribute, remix, adapt, build upon this work non-commercially, and license their derivative works on different terms, provided the original work is properly cited, appropriate credit is given, any changes made indicated, and the use is non-commercial. See: http://creativecommons.org/licenses/by-nc/4.0/.

ORCID iD

Felix J Hüttner http://orcid.org/0000-0002-2299-964X

\section{REFERENCES}

1 Ferlay J, Ervik M, Lam F, et al. Global cancer observatory: cancer today. Lyon, France: international agency for research on cancer, 2018. Available: https://gco.iarc.fr/today accessed 13-OCT-2019

2 Arnold M, Rutherford M, Lam F. Soerjomataram I. ICBP SURVMARK-2 online tool: International cancer survival benchmarking. Lyon, France: International agency for research on cancer, 2019. http://gco.iarc.fr/survival/survmark

3 Ferlay J, Ervik M, Lam F, et al. Global cancer observatory: cancer tomorrow. Lyon, France: International Agency for Research on Cancer, 2018. https://gco.iarc.fr/tomorrow

4 Kamisawa T, Wood LD, Itoi T, et al. Pancreatic cancer. Lancet 2016;388:73-85.

5 Conroy T, Hammel P, Hebbar M, et al. Folfirinox or gemcitabine as adjuvant therapy for pancreatic cancer. $N$ Engl $\mathrm{J}$ Med 2018;379:2395-406.

6 Hiller JG, Perry NJ, Poulogiannis G, et al. Perioperative events influence cancer recurrence risk after surgery. Nat Rev Clin Oncol 2018;15:205-18.

7 Cole SW, Nagaraja AS, Lutgendorf SK, et al. Sympathetic nervous system regulation of the tumour microenvironment. Nat Rev Cancer 2015;15:563-72.

8 Horowitz M, Neeman E, Sharon E, et al. Exploiting the critical perioperative period to improve long-term cancer outcomes. Nat Rev Clin Oncol 2015;12:213-26.
9 Wang D, Dubois RN. Eicosanoids and cancer. Nat Rev Cancer 2010;10:181-93.

10 Mao Y, Sarhan D, Steven A, et al. Inhibition of tumor-derived prostaglandin-e2 blocks the induction of myeloid-derived suppressor cells and recovers natural killer cell activity. Clin Cancer Res 2014;20:4096-106.

11 Zelenay S, van der Veen AG, Böttcher JP, et al. Cyclooxygenasedependent tumor growth through evasion of immunity. Cell 2015;162:1257-70.

12 Karnezis T, Shayan R, Caesar C, et al. VEGF-D promotes tumor metastasis by regulating prostaglandins produced by the collecting lymphatic endothelium. Cancer Cell 2012;21:181-95.

13 Kumar P, Shen Q, Pivetti CD, et al. Molecular mechanisms of endothelial hyperpermeability: implications in inflammation. Expert Rev Mol Med 2009:11:e19.

14 Le CP, Nowell CJ, Kim-Fuchs C, et al. Chronic stress in mice remodels lymph vasculature to promote tumour cell dissemination. Nat Commun 2016;7:10634.

15 Bertolini F, Sukhatme VP, Bouche G. Drug repurposing in oncologypatient and health systems opportunities. Nat Rev Clin Oncol 2015;12:732-42.

16 Pantziarka P, Verbaanderd C, Sukhatme V, et al. ReDO_DB: the repurposing drugs in oncology database. Ecancermedicalscience 2018;12:886.

17 Ricon I, Hanalis-Miller T, Haldar R, et al. Perioperative biobehavioral interventions to prevent cancer recurrence through combined inhibition of $\beta$-adrenergic and cyclooxygenase 2 signaling. Cancer 2019;125:45-56.

18 Sorski L, Melamed R, Matzner P, et al. Reducing liver metastases of colon cancer in the context of extensive and minor surgeries through $\beta$-adrenoceptors blockade and COX2 inhibition. Brain Behav Immun 2016;58:91-8.

19 Haldar R, Ricon-Becker I, Radin A, et al. Perioperative COX2 and $\beta$-adrenergic blockade improves biomarkers of tumor metastasis, immunity, and inflammation in colorectal cancer: A randomized controlled trial. Cancer 2020;126:3991-4001.

20 Chan A-W, Tetzlaff JM, Altman DG, et al. SPIRIT 2013 statement: defining standard protocol items for clinical trials. Ann Intern Med 2013;158:200-7.

21 Schneider M, Strobel O, Hackert T, et al. Pancreatic resection for cancer-the Heidelberg technique. Langenbecks Arch Surg 2019;404:1017-22.

22 Bhattacharyya GS, Babu KG, Bondarde SA, et al. Effect of coadministered beta blocker and COX-2 inhibitor to patients with pancreatic cancer prior to receiving albumin-bound (NAB) paclitaxel. JCO 2015;33:302.

23 Bassi C, Marchegiani G, Dervenis C, et al. The 2016 update of the International Study Group (ISGPS) definition and grading of postoperative pancreatic fistula: 11 years after. Surgery 2017;161:584-91.

24 Wente MN, Bassi C, Dervenis C, et al. Delayed gastric emptying (DGE) after pancreatic surgery: a suggested definition by the International Study group of pancreatic surgery (ISGPS). Surgery 2007;142:761-8.

25 Wente MN, Veit JA, Bassi C, et al. Postpancreatectomy hemorrhage (PPH): an international Study group of pancreatic surgery (ISGPS) definition. Surgery 2007;142:20-5.

26 Koch M, Garden OJ, Padbury R, et al. Bile leakage after hepatobiliary and pancreatic surgery: a definition and grading of severity by the International Study group of liver surgery. Surgery 2011;149:680-8.

27 Schulz KF, Altman DG, Moher D, et al. Statement: updated guidelines for reporting parallel group randomised trials. BMJ 2010;2010:c332.

28 World Medical Association. World Medical association declaration of Helsinki: ethical principles for medical research involving human subjects. JAMA 2013;310:2191-4.

29 Klotz R, Doerr-Harim C, Ahmed A, et al. Top ten research priorities for pancreatic cancer therapy. Lancet Oncol 2020;21:e295-6. 